\title{
Identification of deformation using invariant surface information
}

\author{
David M. Cash ${ }^{a}$, Tuhin K. Sinha ${ }^{a}$, Chun-Cheng Chen $^{a}$, Benoit M. Dawant ${ }^{b}$, William C. \\ Chapman $^{c}$, Michael I. Miga ${ }^{a}$, Robert L. Galloway ${ }^{a}$ \\ ${ }^{a}$ Vanderbilt University, Dept. of Biomedical Engineering, Nashville, TN, USA \\ ${ }^{b}$ Vanderbilt University, Dept. of Electrical Engineering, Nashville, TN, USA \\ ${ }^{c}$ Washington University School of Medicine, Department of Surgery, St. Louis, MO, USA
}

\begin{abstract}
To compensate for soft-tissue deformation during image-guided surgical procedures, non-rigid methods are often used as compensation. However, most of these algorithms first implement a rigid registration to provide an initial alignment. In liver tumor resections, the organ is deformed on a large scale, causing visual shape change on the organ. Unlike neurosurgery, there is no rigid reference available, so the initial rigid alignment is based on the organ surface. Any deformation present might lead to misalignment of non-deformed areas. This study attempts to develop a technique for the identification of organ deformation and its separation from the problem of rigid alignment. The basic premise is to identify areas of the surface that are minimally deformed and use only these regions for a rigid registration. To that end, two methods were developed. First, the observation is made that deformations of this scale cause noticeable changes in measurements based on differential geometry, such as surface normals and curvature. Since these values are sensitive to noise, smooth surfaces were tesselated from point cloud representations. The second approach was to develop a cost function which rewarded large regions with low closest point distances. Experiments were performed using analytic and phantom data, acquiring surface data both before and after deformation. Multiple registration trials were performed by randomly perturbing the post-deformed surface from a ground truth position. After registration, subsurface target positions were compared with those of the ground truth. While the curvature-based algorithm was successful with analytic data, it could not identify enough significant changes in the surface to be useful for phantom data. The minimal distance algorithm proved much more effective in separating the registration, providing significantly improved error measurements for subsurface targets throughout the whole surface.
\end{abstract}

Keywords: Iterative Closest Point algorithm, curvature, differential geometry, surface fitting, B-splines, radial basis functions, tissue deformation

\section{INTRODUCTION}

Image-guided surgery systems attempt to provide an accurate mapping between the present surgical position and high resolution preoperative tomographic images. Anatomical landmarks or extrinsic fiducials attached to rigid structures in the body are often used to provide the mapping, or registration, between the preoperative image space and the intraoperative location. In the case of liver tumor resection, identifying rigid pointbased landmarks can not be performed with any degree of confidence required for the accuracy necessary in the procedure. The feature most often used is the surface of the liver itself. Intraoperative surface data can be acquired using intraoperative tomography,$^{1}$ contact with optically localized probes ${ }^{2}$ laser range scanning, ${ }^{3,4}$ or ultrasound. ${ }^{5,6}$ Traditional rigid registration techniques, such as various closed-form solutions to the orthogonal

\footnotetext{
Correspondence:

David M. Cash

Department of Biomedical Engineering

Vanderbilt University

P.O. Box 351631, Station B

Nashville, TN 37235

david.m.cash@vanderbilt.edu
} 
procrustes problem ${ }^{7-9}$ or the Iterative Closest Point (ICP) algorithm, ${ }^{10}$ attempt to minimize a global error metric based on distance between the two feature sets.

One of the principle assumptions that has often been made is that the registration between these two spaces is rigid, and that the organ acts as a rigid body. However, many studies proved that significant soft tissue deformation is observed in neurosurgical procedures, ${ }^{11-15}$ where the phenomenon is referred to as brain shift. In other types of procedures, the circumstances surrounding organ deformation are quite different. For example, during hepatic tumor resection cases, the liver is disconnected from restraining ligaments and frequently manipulated and repositioned by the surgeon. This results in deformations which are far less subtle than in neurosurgery. They are observed as a bulk shape change that is visible to the human eye.

Part of the observed differences in intraoperative deformation between the brain and liver are due to the amount of rigid constraints present. The skull serves as a rigid frame of reference, and bony landmarks or external fiducials attached to the cranium serve to provide a reliable rigid registration that aids in quantifying the deformation present in the soft brain tissue. If deformation is present within a feature set, rigid registration algorithms that use a global distance error metric will interpret the deformation as rigid registration error. In attempting to minimize the mean error metric across the entire surface, non-deformed areas will become misaligned. This effect can be observed in Figure 1. Since there is no rigid basis during open abdominal liver procedures, determining a rigid registration that will aid in quantifying deformation proves to be quite challenging.
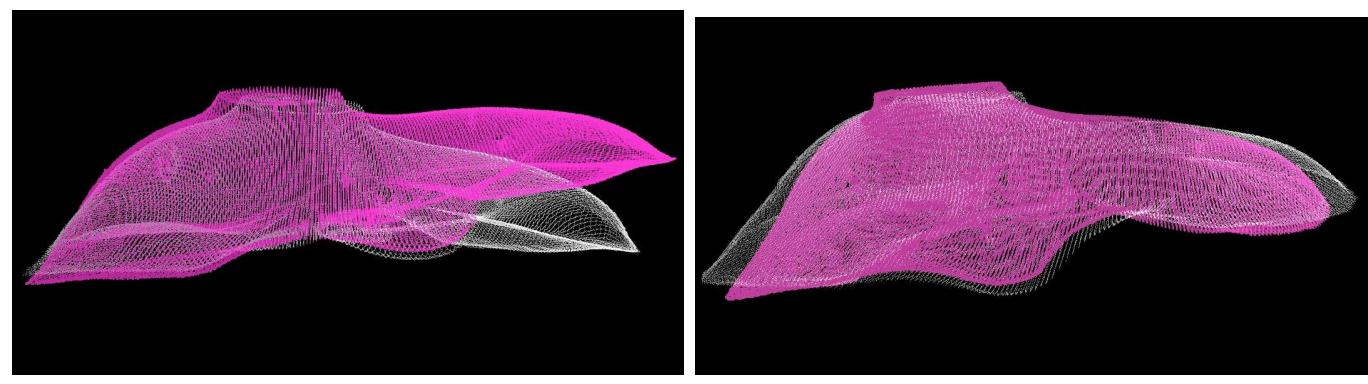

Figure 1. When a deformation is present (left), rigid registration procedures, such as ICP, will attempt to minimize the global error metric, causing some non-deformed areas to become misregistered (right).

There has been some effort into determining non-rigid estimates of deformation based on geometrical features. Chui and Rangarajan developed a technique based on Robust Point Matching (RPM) and the thin plate spline transform. ${ }^{16}$ The technique alternates between constructing a fuzzy correspondence matrix to determine a thin plate spline transform and then using this thin plate spline transform to update the correspondence matrix. Using a deterministic annealing framework, the correspondence matrix iteratively approaches a one-to-one correspondence, with the allowance for some points to be outliers. Other studies have focused on using differential geometry to estimate the deformation. Laskov and Kambhamettu ${ }^{17}$ use an affine motion model which is obtained by minimizing a linear set of equations based on the change of Gaussian curvature to estimate non-rigid motion. The study relied on the critical assumption that the parametrization of their surface was orthogonal at all points on the surface. This method extends upon a similar algorithm used to estimate non-rigid motion using the unit normals of a surface. ${ }^{18}$ Shi et al. ${ }^{19}$ focused on using the principal curvatures to calculate bending energy, which was then used to determine correspondence and define a deformation field. This method was used to track motion of the left ventricular wall.

Rather than estimating the deformation through a set of non-rigid registrations, this study attempts to provide a rigid registration that identifies soft-tissue deformation in the liver and eliminate it from the rigid registration process. An accurate representation of the deformation will be used as input for an algorithm to compensate for the deformation. 


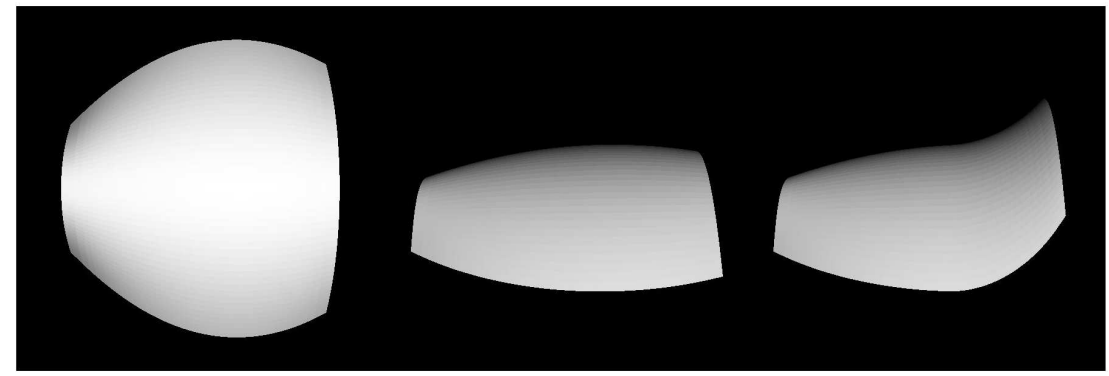

Figure 2. Analytic Surface, the deformation is applied to the right side of the surface, as shown in the bottom image.

\section{METHODS}

\subsection{Overview}

As briefly stated in the Introduction, the goal of this research is to develop a rigid registration that identifies and excludes deformation. Before explaining the methods use to develop these registrations, it is necessary to state some of the assumptions regarding image-guided liver surgery. From the authors' experience observing procedures in the operating room the liver is assumed to be an elastic substance. Unlike neurosurgery, there do not seem to be any apparent fluid effects in the organ, so there is no shrinking or swelling and volume is being preserved. The most obvious feature of the deformation appears to be a shape change in one area, while other regions remain minimally deformed compared to preoperative surface data.

Two means of delineating between deformed and non-deformed surfaces were developed. One method attempted to use differences in curvature between the surfaces to drive the rigid registration. The second method focuses on rewarding registrations in which areas that are minimally deformed are aligned as close as possible, while ignoring the large distances caused by deformation. To that end, a cost function based on the closest point distance operator was developed.

Input data for these two methods consists of three-dimensional point sets. One data set represented a segmented surface from a non-deformed preoperative tomographic image volume. It was registered with another data set representing an intraoperative range scan of the exposed liver surface undergoing deformation. Experiments were performed using analytic and phantom data. The synthetic surfaces were defined through a parametric equation. The points were perturbed by a anisotropic Gaussian noise, which had a higher standard deviation along one axis to mimic the fact that most error in range scanning is found along the depth axis. The deformation was artificially generated on the right side by adjusting the point positions. The surface and subsequent deformation are shown in in figure 2. Phantom data was acquired from a silicone model of the human liver. The phantom was attached to a plexiglass base, and then an object was placed underneath the liver on the base, inducing deformations on the order of $4-5 \mathrm{~cm}$. Corresponding tomogram slices before and after deformation are displayed in figure 1, and a three-dimensional visualization of the resulting deformation is provided in the left image of figure 3 .

\subsection{Identifying deformation using curvature}

Curvature is a differential geometry term that is popular in computer vision because of its invariance to transformation. Despite the orientation of the surface, the values of the principal curvatures do not change. A surface $S$ can often be represented in parametric coordinates, $u$ and $v$, which at any point in the surface are aligned within a plane tangent to the point. The surface normal can be defined in terms of these two orthogonal bases,

$$
\hat{\mathbf{n}}=\frac{S_{u} \times S_{v}}{\left|S_{u} \times S_{v}\right|}
$$

where $S_{u}$ and $S_{v}$ are the derivatives in the $u$ and $v$ directions respectively. For any surface, two other terms, defined as the two fundamental forms can also be defined,

$$
I(u, v)=\left(\begin{array}{cc}
S_{u} S_{u} & S_{u} S_{v} \\
S_{u} S_{v} & S_{v} S_{v}
\end{array}\right)=\left(\begin{array}{cc}
E & F \\
F & G
\end{array}\right)
$$




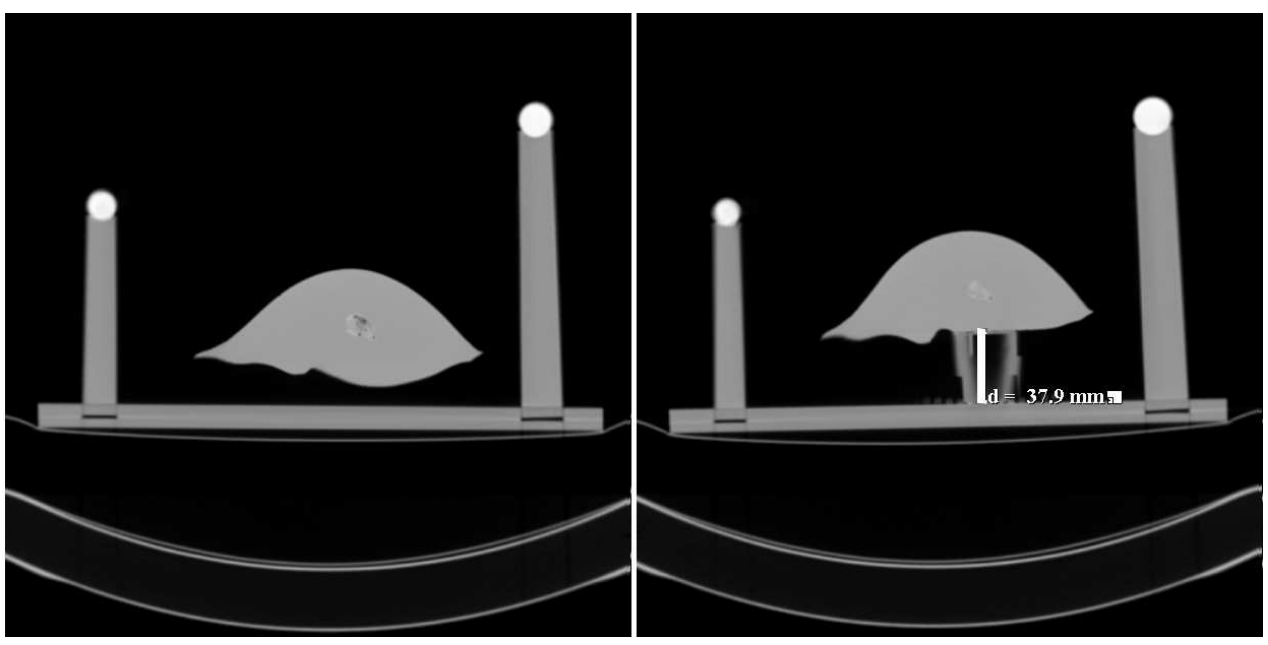

Figure 3. Corresponding CT slices from deformed and non-deformed image. The plastic object placed below the liver produces the deformation observed in Figure 1. At this point in the volume, there is a displacement of $37.9 \mathrm{~mm}$ by the object
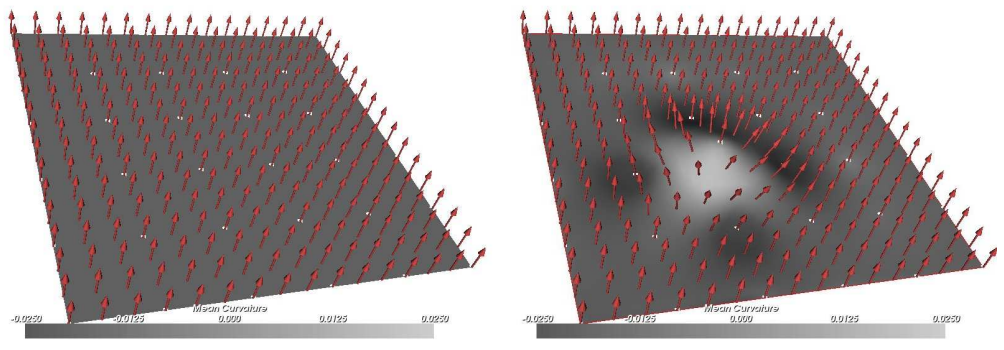

Figure 4. The relationship between deformation, surface normals, and curvature. Away from the deformation, the curvature is unaffected by the normals.

$$
I I(u, v)=\left(\begin{array}{cc}
S_{u u} \hat{n} & S_{u v} \hat{n} \\
S_{u v} \hat{n} & S_{v v} \hat{n}
\end{array}\right)=\left(\begin{array}{cc}
L & M \\
M & N
\end{array}\right)
$$

Gaussian $(K)$ and mean $(H)$ curvature can be expressed in terms of the two fundamental forms.

$$
\begin{aligned}
& K=\operatorname{det}\left(\left(\begin{array}{ll}
E & F \\
F & G
\end{array}\right)^{-1}\left(\begin{array}{cc}
L & M \\
M & N
\end{array}\right)\right) \\
& H=\frac{1}{2} \operatorname{tr}\left(\left(\begin{array}{ll}
E & F \\
F & G
\end{array}\right)^{-1}\left(\begin{array}{cc}
L & M \\
M & N
\end{array}\right)\right)
\end{aligned}
$$

Curvature can be used to represent changes in the surface as shown in figure 4. The left column represents a non-deformed plane with arrows representing the normals. As can be expected, the orientation of the surface normals as well as the value of curvature are uniform. In the right column, a deformation has been added by using a thin plate spline transform. At the area of deformation, a significant change has occurred to both the surface normals and the curvature.

Before curvature values could be calculated for the input point sets, the underlying surface that is represented by the data must be determined. Two different techniques were used to tessellate these surfaces. The first method was a least-squares fit of the point set to a B-spline. The most sensitive part of this algorithm is determining 
the parametrization for each point to be fit. A method similar to one described by Ma and Kruth ${ }^{20}$ has been implemented. Another method incorporates Radial Basis Functions(RBF) to fit the data. A biharmonic RBF was used to interpolate the signed distance for any point from the surface, resulting in the surface being described by the zero isocontour of the RBF. To make this method computationally efficient for large data sets, a special implementation which provides for the fast evaluation and solution of RBFs was used, ${ }^{21}$ developed by FarField Technology (FastRBF, Far Field Technology, Christchurch NZ).

From the underlying surface descriptions, the curvature was then calculated. At each point of the surface, curvature was approximated by fitting a quadric to the local area around the point. ${ }^{22}$ The quadric surface has the parametric representation,

$$
S(x, y, z) \Rightarrow S(u, v)=\left(u, v, a u^{2}+2 b u v+c v^{2}\right)
$$

For each surface fit the third coordinate is aligned with the surface normal of the origin point, while the first and second coordinates are orthogonal bases in the tangent plane. Every surrounding point included in the fit is projected into the tangent plane along the surface normal. The projection point on the tangent plane along with the the projection distance along the normal to the plane are used for the values of the least squares fit. The resulting parameters used to describe the quadric fit can also be used to determine gaussian and mean curvature,

$$
K=4 a c-b^{2}, H=a+c
$$

The number of points included in each quadric fit was based on the number of edges between each candidate point and the origin point for the fit. As the connectivity level was increased, more points were included in to the local quadric fit. The first test performed was analyzing the difference in curvatures between the pre-deformed and post-deformed surface. Since deformation can hinder the ability to achieve proper correspondence using the closest point operator, a different method of correspondence was necessary. Since both data sets were fit in the same manner over similar extents, correspondence was based on the parametric coordinates of the fitted surface.

\subsection{Identifying deformation using the closest point operator}

An alternate approach developed to identify deformation was based on using the closest point distance operator. In regions where the surface is minimally deformed, correct alignments will result in closest point distances close to zero while areas where significant deformation is present will have much higher closest point distances. To take advantage of a large region with low closest point distances, the operator was modified to produce the following distance function.

$$
f(S)=\sum_{i=1}^{N_{S}} \exp \left(-\left|d_{i}\right| / \tau\right)
$$

As more points approach a closest point distance of zero, the cost function will become greater. At the same time, this cost function will not punish points which have large closest point distances due to deformation. The amount of tolerance that is given to the effect of distances is controlled by the spatial constant $\tau$. Currently, the cost function is optimized with respect to a rigid transformation with six degrees of freedom using Powell's method. ${ }^{23}$

Trials were performed on the analytic and phantom surfaces. In both data sets, a ground truth registration was known. For the phantom data, the ground truth was acquired by a point-based registration on external fiducials surrounding the phantom. Random perturbations of the data were generated using transformations in which the six degrees of freedom were randomly selected from $\pm 10 \mathrm{~mm}$ in translation and $\pm 10^{\circ}$ for the rotation. Registrations were also calculated using Iterative Closest Point by means of a comparison. Error measurements were made by comparing registration results with the ground truth. For the analytic surface, this value was the RMS distance between corresponding deformed points after the registration and at ground truth. For the phantom data, the location of six subsurface targets representing tumors were used. 


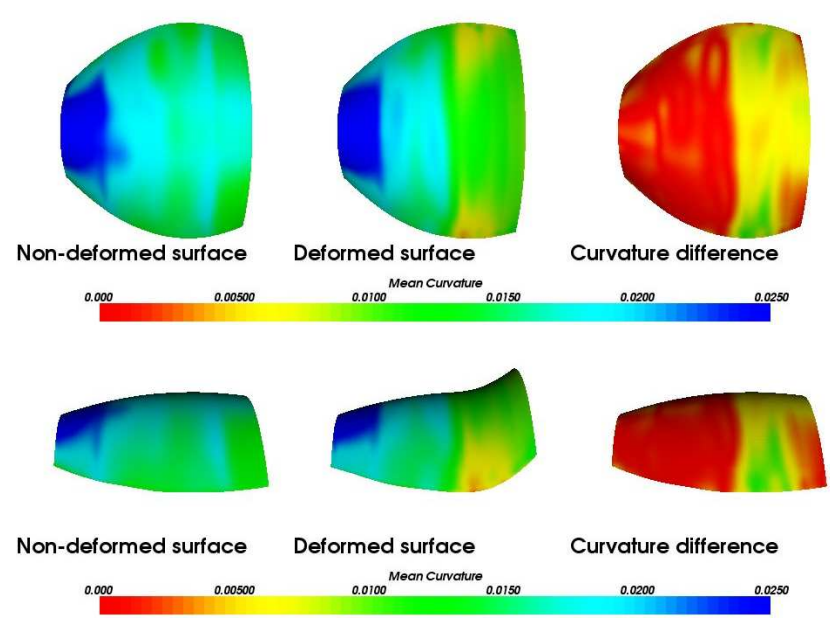

Figure 5. Difference in curvature, analytic surfaces. Full color figures can be found at www.tgt.vanderbilt.edu. In greyscale, high curvature values are on the left side of the first two surfaces, and dark grey regions of the difference image map to low values.

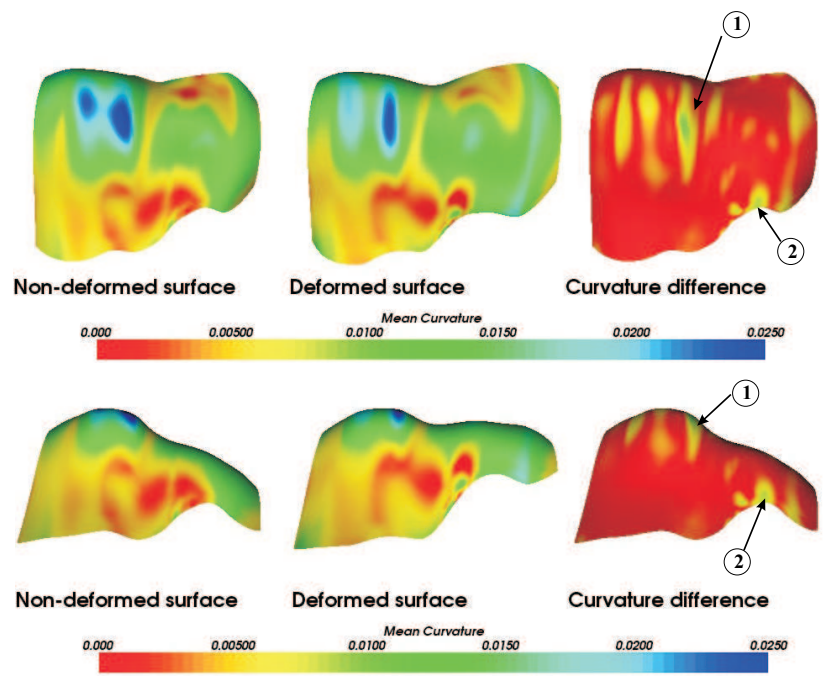

Figure 6. Difference in curvature, phantom surfaces. Full color figures can be found at www.tgt.vanderbilt.edu. In greyscale, high curvature values are at the knob area on top of the phantom, and dark grey regions of the difference image map to low values. Numbered labels indicate where curvature differences correspond to deformation.

\section{RESULTS}

Figure 5 and 6 present results of using curvature to identify deformation. The first two columns show the non-deformed and deformed surfaces with the color of each point based on the curvature value. The third column represents the non-deformed surface as well, except that the color now represents the difference between curvature from each corresponding point pair. In both cases the right side of the surface was deformed to simulate the typical nonrigid behavior of the left lobe that that is observed during surgery. The deformed surfaces were aligned using a pre-determined ground truth. In the case of the synthetic surface, the non-deformed regions of the pre and post-deformation data sets were already aligned. For the phantom, the ground truth was based on external teflon spheres that served as rigid fiducials for a point based algorithm. Using $n=7$ points, the FRE was $0.1 \mathrm{~mm}$, providing an excellent rigid reference between the data. Once ground truth alignments were performed, correspondence between the non-deformed and deformed data set was established through using the identical parametric coordinates from the spline fit. 


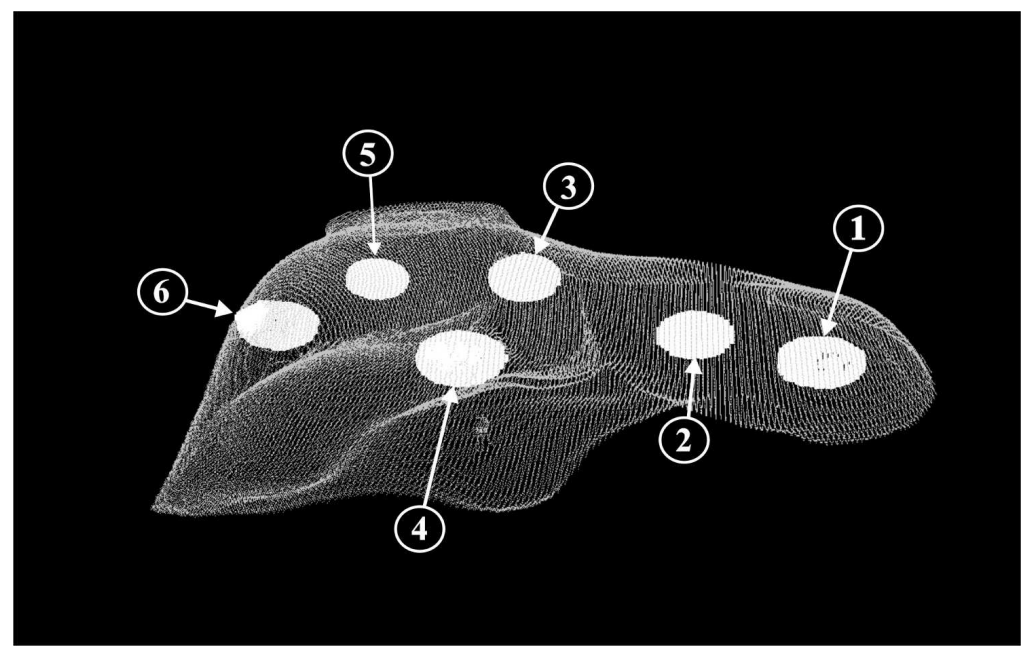

Figure 7. Liver phantom with subsurface targets labelled for use in table 1

\begin{tabular}{|c|c|c|c|c|c|c|c|}
\hline Method & Trials & Tumor 1 & Tumor 2 & Tumor 3 & Tumor 4 & Tumor 5 & Tumor 6 \\
\hline ICP & 2000 & 35.1 & 24.0 & 10.9 & 9.1 & 19.1 & 21.6 \\
Closest Point - Success & 1886 & 16.6 & 13.2 & 8.1 & 7.4 & 2.3 & 5.7 \\
Closest Point - Failure & 114 & 36.4 & 28.4 & 16.4 & 12.6 & 14.0 & 16.6 \\
\hline
\end{tabular}

Table 1. RMS distance, mm, between tumor locations after registration and the ideal location given by the ground truth

Using the cost function which is based on the closest point distance operator, registrations were performed on the predeformed and post-deformed analytic surface. After 1000 trials, the RMS distance between points on the deformed surface after the registration compared to their corresponding ground truth was $0.40 \mathrm{~mm}$, with the maximum RMS distance being $1.03 \mathrm{~mm}$. On the other hand, the Iterative Closest Point method for the same set of perturbations resulted with an average RMS distance of the 1000 trials being $3.74 \mathrm{~mm}$, with a maximum RMS distance of any trial being $4.20 \mathrm{~mm}$.

Table 1 shows results for the registration studies using the liver phantom. Here the RMS distance between the location at the six tumor targets at ground truth and their position after registration are shown. The location of the tumors with respect to the liver surface is shown in 7 .

The results from registration based on modifying the closest point distance operator are divided into to two categories: "success" and "failure". A registration was deemed a success if it qualitatively captured the observed deformation and had error distances significantly lower than found from the ICP method. The success trials also converged to approximately the same point, which corresponds to the lowest minima observed in simulations.

\section{DISCUSSION}

While curvature metrics have definite potential in the identification of deformed regions, there are still some significant issues. In figure 5, the values of curvature have significantly changed after being deformed. The difference in curvature clearly highlights the boundary of the deformation. However, the curvature data of the phantom surface in figure 6 is more ambiguous. There are some clear instances where deformation and curvature coincide, which are labelled regions "1" and "2". Region 1 is located at the top of the liver, where there is an inflection point between the deformation and the fixed region. Region 2 resides on the lower front side of the liver right at the ridge. While both of these represent areas of deformation, there are still many other portions of the deformed left lobe which do not show significant changes in curvature.

Curvature is still considered by the authors to be a valuable metric for the purpose of identifying deformation, although there are some issues to resolve. As shown in (4) and (5), this metric is based on the second-order derivatives of the surface geometry. Noise plays a considerable factor in the resulting curvature values, as does 
the method to calculate curvature on surfaces defined by discrete geometric locations. Many different methods have been proposed for calculating curvature on tesselated surfaces used in computer graphics. The method used in this paper, which fits the local surface of each point to a quadric, has been used quite often. Batchelor et al. ${ }^{24}$ proposed a method to directly calculate the curvature using the three-dimensional topology as described by the point of interest, its surrounding points, and the connecting edges. This method produces satisfactory results as long as the aspect ratio and connectivity of the tessellating polygons is of good quality. Others have gone in the opposite direction, using whole three-dimensional volumes that depict the surface as an isocontour. ${ }^{25}$ If the isocontour is well-behaved, then the surface normal can be related to the image's intensity gradient, and curvature can be determined based on the derivatives of the pixel's intensity values. While this method has the potential to offer the smoothest interpretation of curvature, it also requires making nine derivative calculations for every point for which curvature needs to be obtained.

All of the above methods differ in the information used to represent the surface and calculate the corresponding curvature, yet all are dependent on the amount of surrounding geometric data that is included in the calculation. Using a similar method of calculation, Shi's study performed an analysis on the behavior curvature with respect to the extent of surrounding surface information used in the calculation. ${ }^{19}$ By incorporating only points directly connected to the point of interest, curvature is only determined over a small scale. Tiny variations due to noise have significantly more effect. Conversely as more points are included in the calculation, the measurement is less susceptible to noise, but it also is less able to discern specific details in the surface. The results from their study noted that the most significant curvature features of a surface were essentially invariant of the extent of the neighborhood.

The central challenge of incorporating curvature information into the registration process involves determining correspondence between points on the deformed and non-deformed surface. The most common form of determining correspondence is based on the closest point distance operator. For most surfaces and correspondence strategies, closest point distances are used to set up a temporary and arbitrary correspondence, allowing the iterative alignment of images to naturally bring points to their true one-to-one correspondence. With the presence of deformation, the closest point may not be the same as the corresponding point on the deformed surface. As more deformation is present, the less likely that true correspondence will be established through the closest point operator, even when aligned at ground truth. Many groups have proposed modifications to the closest point operator in order to achieve a more accurate correspondence matrix. Auxiliary information about a point or surface, including color, ${ }^{26}$ surface normals and other invariant information based on differential geometry, ${ }^{27,28}$ have all been used to further constrain the closest point distance metric. Others have used this extra information to constrain the search region allowed for corresponding points. ${ }^{19,29}$ Some treat correspondence as a continuously valued problem, allowing fractional correspondence to many points at one time. ${ }^{16}$

While traditional closest point methods may have trouble with large deformation, the operator itself still has valuable information for this problem. Using the closest point distance operator to drive the exponential sum found in the cost function (8), transformations that align data sets such that there are many points with low closest point distances are rewarded. This scenario is associated with minimally deformed regions are wellaligned. At the same time, the cost function does not punish for the large closest point distances associated with deformation. In this set of experiments, the most frequently observed minimum appears to be a global minimum, and most, but not all, of the deformation has been recovered. There is still a failure rate of about $5-6 \%$, so methods for avoiding local minima must be explored.

From the experiments comparing this new cost function with ICP, the most improvement is found at tumors 5 and 6 , where the organ is minimally deformed, yet has been severely misaligned by ICP. Significant improvement is found in tumors 1 and 2, which are the most deformed regions of the liver. While the error for these initial experiments was still over $10 \mathrm{~mm}$, this registration captured a majority of the deformation, which was on the order of $4-5 \mathrm{~cm}$. The least amount of improvement was found at tumors 3 and 4 , which are located near the center of the organ and experience the smallest lever arm effect from the rotation within the ICP transformation.

The results of the rigid registration will be used to provide an accurate representation of the organ deformation, which will serve to construct boundary conditions for a finite element model. Considering the numerous amount of non-rigid registration algorithms available, it might seem more intuitive to implement one of these 
methods instead. In fact, deformable algorithms that use feature and geometric information are being considered in future studies as a means of comparison. However, most of the current deformable algorithms rely on assumptions that may not be found in this situation. While most deformable algorithms may not be based on individual point correspondence, they are based on correspondence of the surface as a whole. In other words, there is an expectation that both data sets are complete representations of the structure. Since the laser range scanner, our most frequent intraoperative data acquisition device, can only acquire information through line of sight, it represents a partial surface compared to the complete organ segmented from preoperative tomography. Even with the inclusion of subsurface structures from ultrasound images, complete organ data cannot be captured during surgery in an expedient or effective manner using sparse data alone. Deformable algorithms must also provide for a manner of accurately determining subsurface deformation. Similar to finite element modelling, this process will likely involve providing boundary conditions for the entire organ surface. Some regions on the undersurface are assumed to be fixed, while other parts of the liver are free to move as dictated by the model. Finite element modelling will be conducted on a complete volumetric mesh based on tomographic studies and is well-suited for constraining regions of the surface not visualized by intraoperative methods.

\section{CONCLUSION}

Two methods for identifying large deformations in anatomical surfaces were developed. One focuses on differences of curvature after a deformation has occurred, the other looks to align the minimally deformed surfaces by using a modification of the closest point operator, which rewards regions of closely aligned surfaces while not punishing large closest point distance operators where deformation is likely present. Unlike traditional landmark based registrations which rely on global distance error metrics, both methods have the ability to identify deformation in surfaces. Issues regarding correspondence, curvature, and optimization must be further examined in order to increase the accuracy and robustness of the algorithms.

\section{ACKNOWLEDGEMENTS}

This work was done under the NIH grant R33-CA91352. Many of the algorithms and visualization tools were developed using the Visualization Toolkit (www.vtk.org). The authors would like to acknowledge FarField Technology for the aid in the use of their surface fitting software based on Radial Basis Functions. The authors would also like to thank the CT technicians at Vanderbilt Medical Center's Radiology department for aid in the acquisition of phantom data.

\section{REFERENCES}

1. M. Ferrant, A. Nabavi, B. Macq, F. Jolesz, R. Kikinis, and S. Warfield, "Registration of 3-d intraoperative $\mathrm{mr}$ images of the brain using a finite-element biomechanical model," IEEE Transactions on Medical Imaging 20(12), pp. 1384-1397, 2001.

2. A. Herline, J. Herring, J. Stefansic, W. Chapman, R. Galloway Jr., and B. Dawant, "Surface registration for use in interactive, image-guided liver surgery," Comput.Aided Surg. 5(1), pp. 11-17, 2000.

3. D. M. Cash, T. K. Sinha, W. C. Chapman, H. Terawaki, B. M. Dawant, R. L. Galloway, and M. I. Miga, "Incorporation of a laser range scanner into image-guided liver surgery: Surface acquisition, registration, and tracking," Medical Physics 30, pp. 1671-1682, jun 2003.

4. M. Audette, K. Siddiqi, F. Ferrie, and T. Peters, "An integrated range-sensing, segmentation and registration framework for the characterization of intra-surgical brain deformations in image-guided surgery," Computer Vision and Image Understanding 89(2-3), pp. 226-251, 2003.

5. A. Gronningsaeter, A. Kleven, S. Ommedal, T. Aarseth, T. Lie, F. Lindseth, T. Lango, and G. Unsgard, "Sonowand, an ultrasound-based neuronavigation system," Neurosurgery 47(6), pp. 1373-1379, 2000.

6. R. Bucholz, D. Yeh, J. Trobaugh, L. McDurmont, C. Sturm, C. Baumann, J. Henderson, A. Levy, and P. Kessman, "The correction of stereotactic inaccuracy caused by brain shift using an intraoperative ultrasound device," CVRMED-MRCAS'97 1205, pp. 459-466, 1997.

7. H. B.K.P., "Closed-form solution of absolute orientation using unit quaternions," J.Opt.Soc.Amer. 4, pp. 629-642, 1987. 
8. P. H. Schonemann, "A generalized solution of the orthogonal procrustes problem," Psychometrika 31, pp. 1-10, 1966.

9. K. Arun, T. Huang, and S. D. Blostein, "Least-squares fitting of 2 3-D point sets," IEEE Transactions on Pattern Analysis and Machine Intelligence 9, pp. 699-700, Sept. 1987.

10. P. J. Besl and N. D. McKay, "A method for registration of 3-d shapes," IEEE Transactions on Pattern Analysis and Machine Intelligence 14(2), pp. 239-256, 1992.

11. D. Roberts, A. Hartov, F. Kennedy, M. Miga, and K. Paulsen, "Intraoperative brain shift and deformation: a quantitative analysis of cortical displacement in 28 cases," Neurosurgery 43, pp. 749-758, Oct 1998.

12. D. Hill, C. Maurer, R. Maciunas, J. Barwise, J. Fitzpatrick, and M. Wang, "Measurement of intraoperative brain surface deformation under a craniotomy," Neurosurgery 43(3), pp. 514-526, 1998.

13. A. Nabavi, P. Black, D. Gering, C. Westin, V. Mehta, R. Pergolizzi, M. Ferrant, S. Warfield, N. Hata, R. Schwartz, W. Wells, R. Kikinis, and F. Jolesz, "Serial intraoperative magnetic resonance imaging of brain shift," Neurosurgery 48(4), pp. 787-797, 2001.

14. C. Nimsky, O. Ganslandt, S. Cerny, P. Hastreiter, G. Greiner, and R. Fahlbusch, "Quantification of, visualization of, and compensation for brain shift using intraoperative magnetic resonance imaging," Neurosurgery 47(5), pp. 1070-1079, 2000.

15. C. R. Maurer, D. L. G. Hill, A. J. Martin, H. Y. Liu, M. McCue, D. Rueckert, D. Lloret, W. A. Hall, R. E. Maxwell, D. J. Hawkes, and C. L. Truwit, "Investigation of intraoperative brain deformation using a 1.5-t interventional mr system: Preliminary results," IEEE Transactions on Medical Imaging 17(5), pp. 817-825, 1998.

16. H. Chui and A. Rangarajan, "A new point matching algorithm for non-rigid registration," Computer Vision and Image Understanding 89, pp. 114-141, 2003.

17. P. Laskov and C. Kambhamettu, "Curvature-based algorithms for nonrigid motion and correspondence estimation," IEEE Trans. Pattern Analysis And Machine Intelligence 25, pp. 1349-1354, Oct. 2003.

18. C. Kambhamettu, D. Goldgof, M. He, and P. Laskov, "3d nonrigid motion analysis under small deformations," Image and Vision Computing 21(3), pp. 229-245, 2003.

19. P. Shi, A. Sinusas, T. Constable, E. Ritman, and S. Duncan, "Point-tracked quantitative analysis of left ventricular surface motion from 3-d image sequences," IEEE Trans. Med. Im. 19(1), pp. 36-50, 2000.

20. W. Ma and J. Kruth, "Parameterization of randomly measured points for least-squares fitting of b-spline curves and surfaces," Computer-Aided Design 27(9), pp. 663-675, 1995.

21. J. Carr, B. Beatson, R.K. adn McCallum, W. Fright, T. McLennan, and T. Mitchell, "Smooth surface reconstruction from noisy range data," in ACM GRAPHITE 2003, pp. 119-126, (Melbourne, Austraila), 2003.

22. E. Stokely and S. Ww, "Surface parameterization and curvature measurement of arbitrary 3-d objects - 5 practical methods," IEEE Transactions on Pattern Analysis and Machine Intelligence 14(8), pp. 833-840, 1992. JAUG.

23. W. H. Press, S. A. Teukolsky, W. T. Vetterling, and B. P. Flannery, Numerical Recipes in C: The Art of Scientific Computing, Cambridge University Press, New York, 2nd ed., 1992.

24. P. Batchelor, A. Smith, D. Hill, D. Hawkes, T. Cox, and A. Dean, "Measures of folding applied to the development of the human fetal brain," IEEE Transactions on Medical Imaging 21(8), pp. 953-965, 2002.

25. O. Monga and S. Benayoun, "Using partial derivatives of 3d images to extract typical surface features," Computer Vision and Image Understanding 61, pp. 171-189, Mar 1995.

26. A. E. Johnson and S. B. Kang, "Registration and integration of textured 3D data," Image and Vision Computing 17, pp. 135-147, Feb. 1999.

27. G. Sharp, S. Lee, and D. Wehe, "Icp registration using invariant features," IEEE Transactions on Pattern Analysis and Machine Intelligence 24(1), pp. 90-102, 2002.

28. D. Meier and E. Fisher, "Parameter space warping: Shape-based correspondence between morphologically different objects," IEEE Transactions on Medical Imaging 21(1), pp. 31-47, 2002.

29. G. Godin, D. Laurendeau, and R. Bergevin, "A method for the registration of attributed range images," 2001. 